\title{
Design and characterization of cyclosporine A-loaded nanofibers for enhanced drug dissolution
}

\author{
Poornima Dubey ${ }^{a}$, Susan A. Barker ${ }^{a, b}$ and Duncan Q.M. Craig ${ }^{a *}$ \\ aUCL School of Pharmacy, 29-39 Brunswick Square, London WC1N 1AX, United Kingdom \\ ${ }^{b}$ Current address - Medway School of Pharmacy, The Universities of Greenwich and Kent at Medway, \\ Anson Building, Kent ME4 4TB, United Kingdom \\ *Corresponding author
}

Email addresses:

p.dubey@ucl.ac.uk (P.Dubey)

s.barker@greenwich.ac.uk (S.A.Barker)

duncan.craig@ucl.ac.uk (D.Q.M. Craig) 
a)

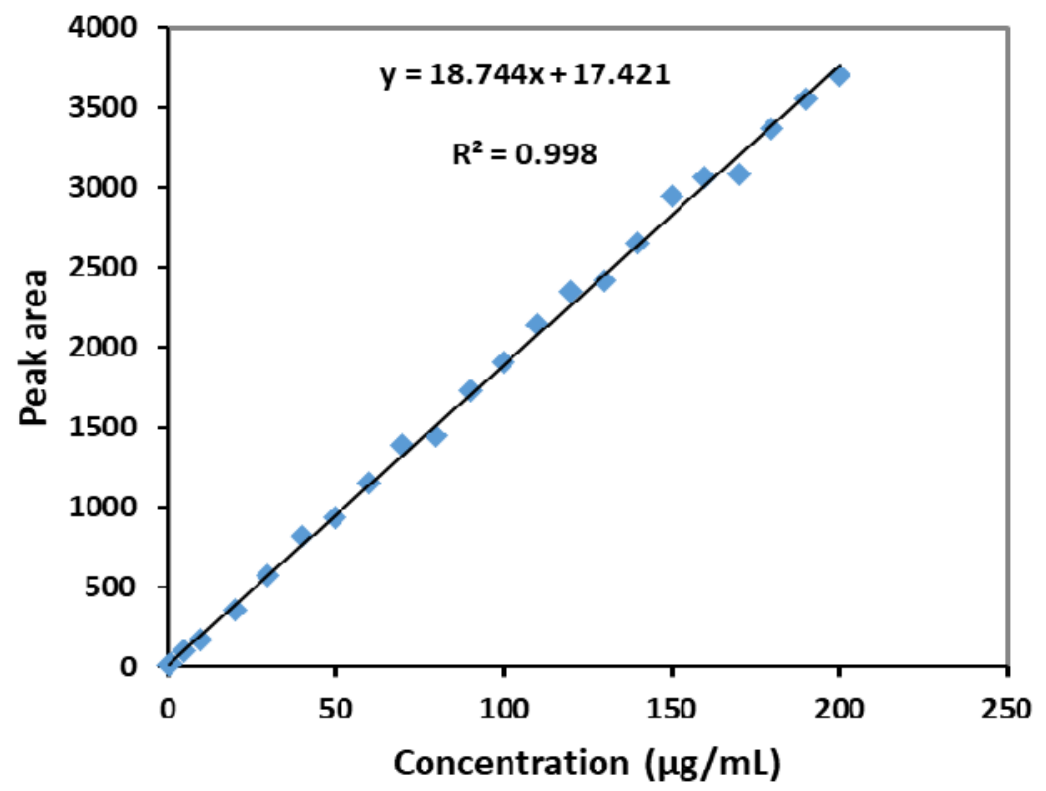

b)

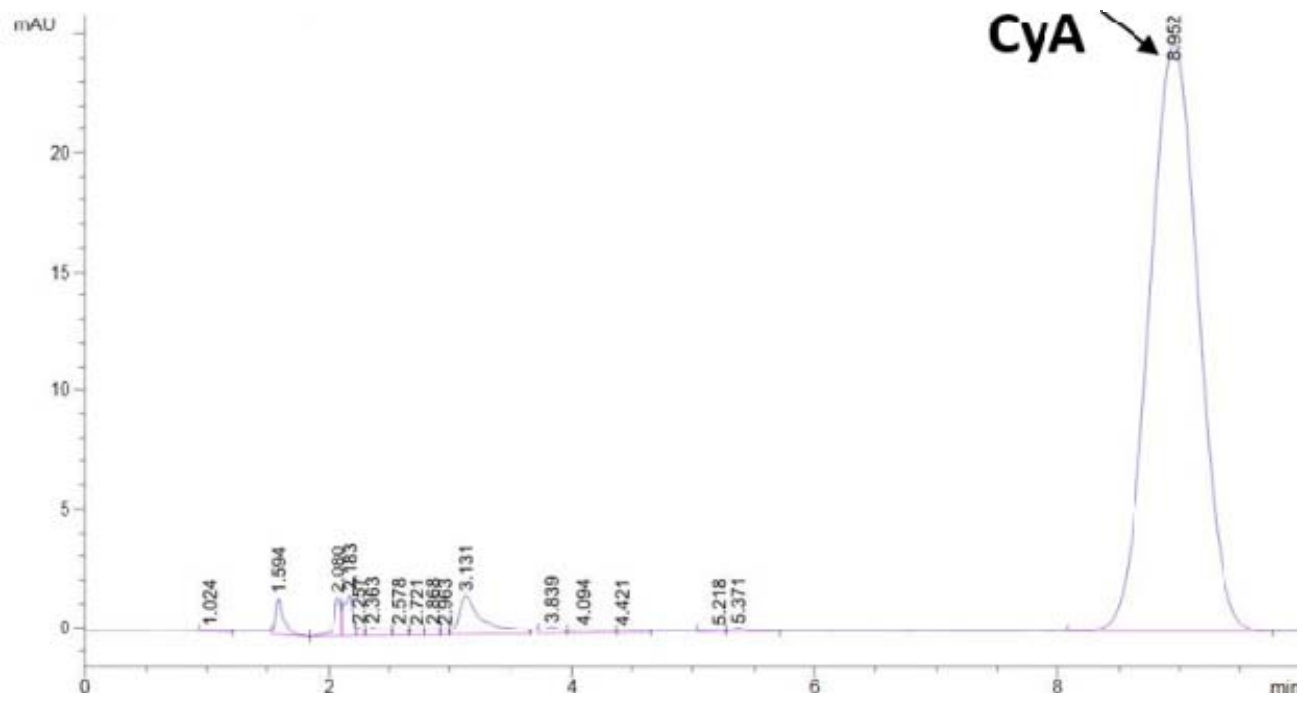

Figure S1: a) Plot of CyA concentration versus peak area obtained by HPLC-UV b) Typical chromatogram for the CYA HPLC-UV analysis at $210 \mathrm{~nm}$ 
a)

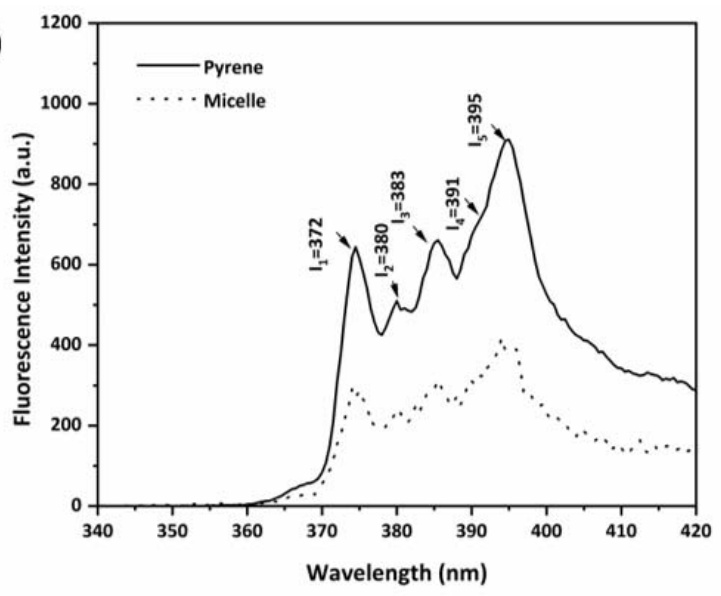

b)

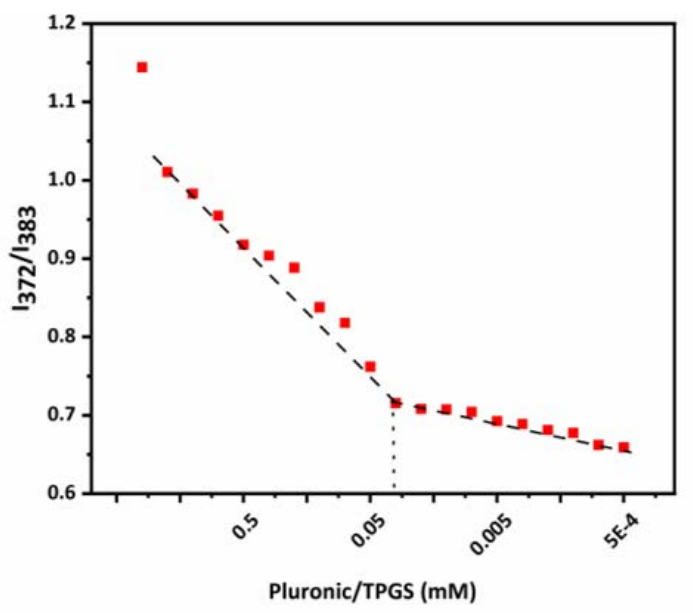

Figure S2: a) Fluorescence spectra of pyrene $(0.6 \mu \mathrm{M})$ as fluorescence hydrophobic probe alone and in micellar suspension (3:2 F127/TPGS) at $25^{\circ} \mathrm{C}$, b) Plot of the fluorescence emission of pyrene at the $1372 / 1383$ ratio in the presence of various concentrations $(0.0005 \mathrm{mM}-0.1 \mathrm{mM})$ of copolymers F127/TPGS (3:2 molar ratio).

\begin{tabular}{|l|l|l|}
\hline Surfactants & Ratios & CMC (mM) \\
\hline TPGS & $1: 0$ & 0.46 \\
\hline F127 & $1: 0$ & 0.093 \\
\hline F127/TPGS & $1: 1$ & 0.13 \\
\hline F127/TPGS & $1: 4$ & 0.023 \\
\hline F127/TPGS & $3: 2$ & 0.062 \\
\hline
\end{tabular}

Table S1: Critical micelle concentrations (mM) of surfactants Pluronic F127 and TPGS alone, and in combination at a range of molar ratios from 1:0, 1:1, 1:4 and 3:2 respectively. 


\begin{tabular}{|l|c|}
\hline Surfactants & Concentration $(\mu \mathrm{g} / \mathrm{mL})$ \\
\hline 10mM F127/TPGS & 933.8 \\
\hline 1mM F127/TPGS & 122.3 \\
\hline 0.1mM F127/TPGS & 73.0 \\
\hline 0.0005mM F127/TPGS & 52.4 \\
\hline Alone PBS & 7.3 \\
\hline PBS+30\% Ethanol & 12.0 \\
\hline PBS+0.5\% Tween & 14.0 \\
\hline
\end{tabular}

Table S2: Equilibrium solubility of CyA in relation to molar concentration $(0.0005 \mathrm{mM}-10 \mathrm{mM})$ of surfactant mixture (F127:TPGS; 3:2 molar ratio) in aqueous solution at $37^{\circ} \mathrm{C}$.

\begin{tabular}{|l|c|c|}
\hline $\begin{array}{l}\text { Functional } \\
\text { groups }\end{array}$ & $\begin{array}{c}\text { Wavenumber }\left(\mathrm{cm}^{-\mathbf{l}}\right) \text { of CyA } \\
\text { Wavenumber }\left(\mathrm{cm}^{-\mathbf{l}}\right) \text { of }\end{array}$ & $\begin{array}{l}\text { Wavi loaded micelle } \\
\text { CyA }\end{array}$ \\
\hline n(C-H) alkyl (s) & $2960,2930,2875$ & 2880 \\
\hline Amine, O-H (b) & 3305 & - \\
\hline C=O (s) & 1290 & 1245 \\
\hline CONH & 1630 & 1625 \\
\hline O-H (b) & 1475 & 1465 \\
\hline C-H2 (as) & 1410 & 1415 \\
\hline C-O-C(s) & 1205 & 1235 \\
\hline C-N (s) & 1295,1255 & 1280,1240 \\
\hline C-O (s) & - & 1340 \\
\hline C-N (s) & 1090 & 1100 \\
\hline C=C (s) & 975 & 965 \\
\hline
\end{tabular}

Table S3: Major vibrational frequencies showed in the IR spectrum of Cyclosporine $A$ alone and on incorporation into 3:2 F127/TPGS micelles (where notation s=symmetric, as=asymmetric and $b=$ bending vibrations). 


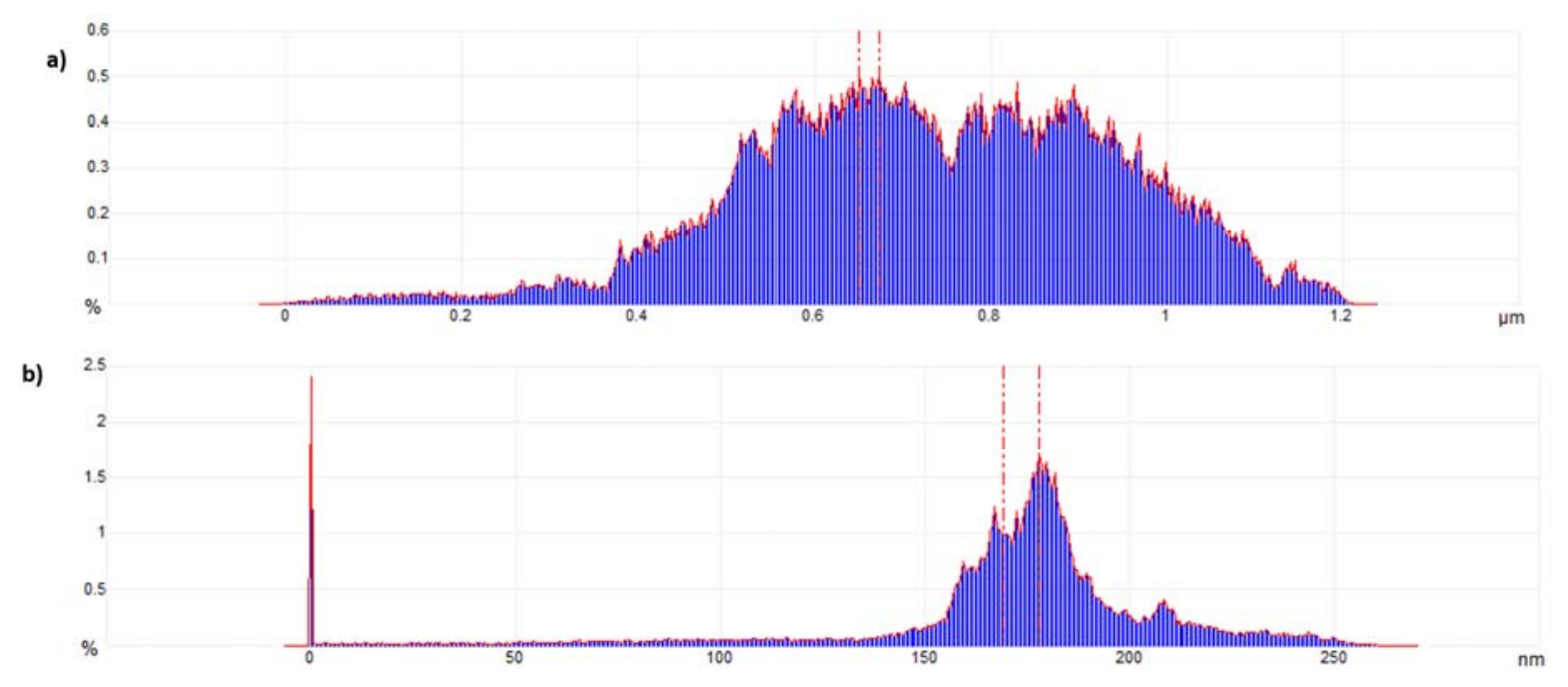

Figure S3: Histogram of a) unloaded PVP nanofibers and b) CyA/micelle loaded nanofibers showing the height distribution for the two sets of samples; analysis performed using AFM Nanoscope analysis software version 1.70.
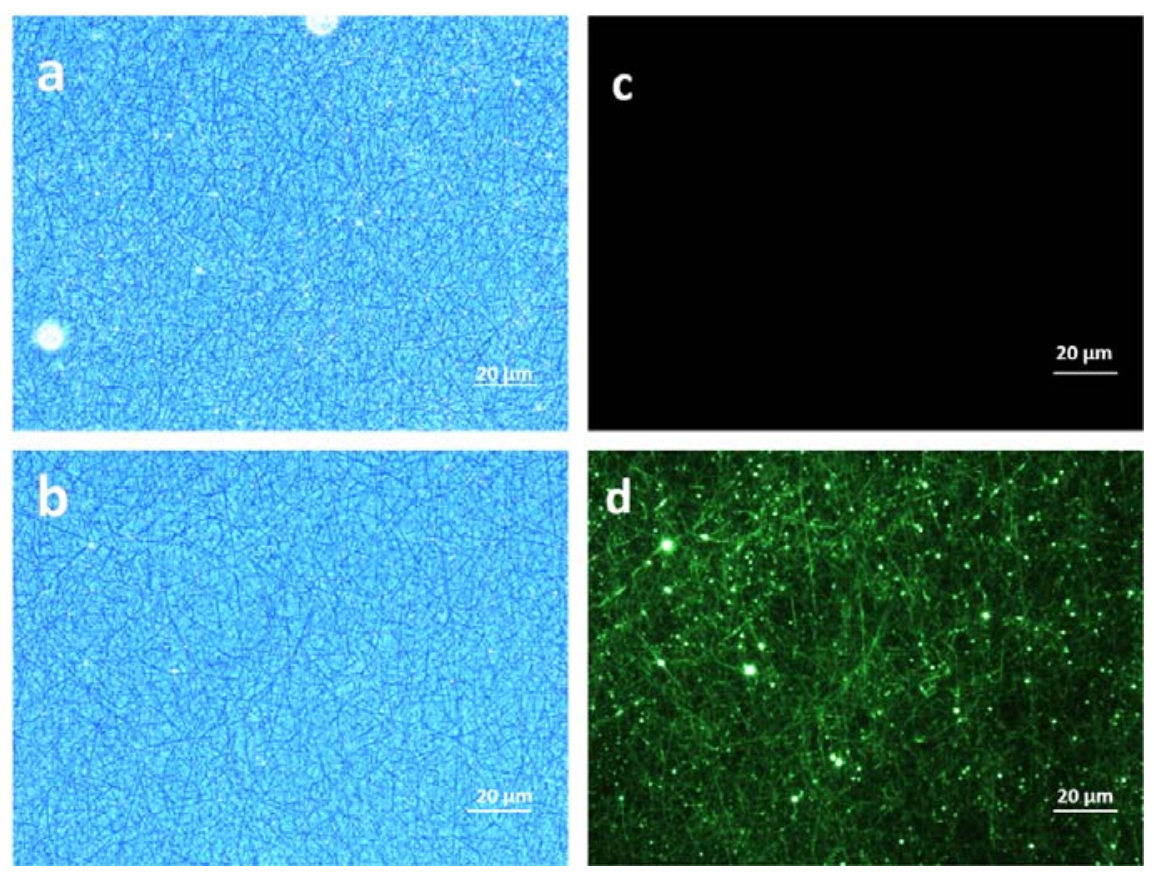

Figure S4: Investigation of micelle loading into nanofibers using fluorescence microscopic imaging: $a, c)$ blank PVP nanofibers under bright field and green fluorescence protein (GFP) filter and $b, d)$ coumarin-6 loaded micelles incorporated into PVP nanofibers under bright field and GFP filters respectively. The images were taken using a 20X objective lens. 


\begin{tabular}{|c|c|c|c|c|c|}
\hline \multirow[t]{2}{*}{ Model } & \multirow[t]{2}{*}{ Parameter } & \multicolumn{3}{|c|}{$\begin{array}{c}\text { CyA micelles loaded nanofiber } \\
\text { Formulation }\end{array}$} & \multirow[t]{2}{*}{ Factors } \\
\hline & & pH 1.2 & pH 7.4 & pH 6.8 & \\
\hline $\begin{array}{l}\text { Zero order } \\
\mathrm{F}_{\mathrm{t}}=\mathrm{k}_{0} \mathrm{t}\end{array}$ & $\begin{array}{l}\mathrm{k}_{0} \\
\mathbf{R}^{2} \_ \text {adj } \\
\text { AIC } \\
\text { MSC }\end{array}$ & $\begin{array}{c}7.6520 \\
\\
\mathbf{0 . 9 8 6 8} \\
\mathbf{7 3 . 1 9 5 1} \\
4.1201\end{array}$ & $\begin{array}{c}8.154 \\
\\
0.9943 \\
\mathbf{6 2 . 9 7 5 7} \\
5.0192\end{array}$ & $\begin{array}{c}8.488 \\
\\
0.9666 \\
86.9356 \\
3.2554\end{array}$ & $\mathrm{k}_{0}$-apparent dissolution rate constant. \\
\hline $\begin{array}{l}\text { Korsmeyer-Peppas } \\
\mathrm{F}=\mathrm{k}_{\mathrm{KP}}{ }^{* t^{\mathrm{n}}}\end{array}$ & $\begin{array}{l}\mathrm{k}_{\mathrm{KP}} \\
\mathrm{n} \\
\mathbf{R}^{2} \text { adj } \\
\mathbf{A I C} \\
\mathrm{MSC}\end{array}$ & $\begin{array}{l}11.366 \\
0.825 \\
0.9942 \\
61.5753 \\
4.9501\end{array}$ & $\begin{array}{c}10.034 \\
0.908 \\
0.9980 \\
49.0621 \\
6.0130\end{array}$ & $\begin{array}{c}13.721 \\
0.785 \\
\\
0.9927 \\
66.5040 \\
4.7148\end{array}$ & $\begin{array}{l}\mathrm{n} \text { - the diffusional exponent indicating the } \\
\text { drug-release } \\
\text { mechanism } \\
\mathrm{k}_{\mathrm{KP}} \text { - the release constant incorporating } \\
\text { structural and } \\
\text { geometric characteristics of the drug- } \\
\text { dosage form }\end{array}$ \\
\hline $\begin{array}{l}\text { Hopfenberg } \\
F=100^{*}\left[1-\left(1-k_{H B}{ }^{*} t\right)^{m}\right]\end{array}$ & $\begin{array}{l}\mathrm{k}_{\mathrm{HB}} \\
\mathrm{n} \\
\mathrm{R}^{2} \_ \text {adj } \\
\text { AIC } \\
\text { MSC }\end{array}$ & $\begin{array}{c}0.062 \\
1.507 \\
\\
\mathbf{0 . 9 9 4 1} \\
\mathbf{6 1 . 9 1 1 9} \\
4.9261\end{array}$ & $\begin{array}{c}\mathbf{0 . 9 9 5 8} \\
\mathbf{5 9 . 3 7 8 7} \\
5.2761\end{array}$ & $\begin{array}{c}0.075 \\
1.372 \\
\\
\mathbf{0 . 9 8 8 0} \\
\mathbf{7 3 . 4 9 4 7} \\
4.2155\end{array}$ & $\begin{array}{l}\text { The combined constant, } \mathrm{k}_{\mathrm{HB}}=\mathrm{k}_{0} /\left(\mathrm{C}_{0} \mathrm{x}\right. \\
\left.\mathrm{a}_{0}\right) \text {; where } \mathrm{k}_{0} \\
\text { is the erosion rate constant, } \mathrm{C}_{0} \text { is the } \\
\text { initial concentration of drug in the } \\
\text { matrix, } \mathrm{a}_{0} \text { is the initial radius for a sphere } \\
\text { or cylinder or the half-thickness for a } \\
\text { slab; } \mathrm{n} \text { is } 1,2 \text {, and } 3 \text { for a slab, cylinder, } \\
\text { and sphere, respectively }\end{array}$ \\
\hline Comparison result & \multicolumn{5}{|c|}{$\begin{array}{l}\text { R2_adj, AIC and MSC of above three model were compared for drug release at different } \mathrm{pH} \text {. All } \\
\text { three model share close } \mathrm{R} 2 \text { adj value for } \mathrm{pH} 7.4 \text { and } 1.2 \text { with difference for } \mathrm{pH} 6.8 \text {. Hence to } \\
\text { understand the best fit model AIC was compared as minimum AIC represents best fit, it was } \\
\text { observed that at all three } \mathrm{pH} \text { AIC was minimum for Korsmeyer-peppas model along with high } \\
\mathrm{R} 2 \text { adj value. Further MSC was compared by all the three methods and it was found that highest } \\
\mathrm{MSC} \text { value were for all the pH with Korsmeyer-peppas model, whereas Hopfenberg model presents } \\
\text { closeness in terms of best fit model dissolution behaviour. Hence based on kinetic studies drug } \\
\text { follows non-fickian diffusion (nearly } \mathrm{n}<0.8 \text { ) along with disintegration of polymeric matrix. }\end{array}$} \\
\hline
\end{tabular}

Table S4: Kinetic models studied for cyclosporine A release from CyA loaded micelle incorporated composite nanofibers at different $\mathrm{pH}$ values using DDSolver software. Table shows the best fit models based on comparison of statistical criteria including the adjusted coefficient of determination (Rsqr_adj or R2 adjusted), the Akaike Information Criterion (AIC), and the Model Selection Criterion (MSC). The three closest fit kinetic models were zero order, KorsmeyerPeppas and Hopfenberg models respectively. 